\title{
Non-linear forced vibration analysis of rectangular plates including the coupling between transverse and in-plane displacements.
}

\author{
E. Merrimi ${ }^{1}$, K. El Bikri ${ }^{1}$, R. Benamar ${ }^{2}$ \\ ${ }^{1}$ Université Mohammed V-Souissi, Ecole Normale Supérieure de l'Enseignement Technique Rabat, LaMIPI, B.P. 6207, \\ Rabat Instituts, Morocco \\ ${ }^{2}$ Ecole Mohammadia d'Ingénieurs, Département des EGT Av. Ibn Sina, Agdal, Rabat, Morocco
}

\begin{abstract}
The objective of this work is to study the geometrically non-linear steady state periodic forced response of fully clamped rectangular plates (FCRP) with immovable in-plane conditions, taking into account the effect of the in-plane displacements. A complete formulation has been proposed first, reducing the equations of motion to a system of coupled non-linear algebraic equations, which are decoupled once the in-plane inertia is omitted. An averaging technique has then been developed, in order to simplify the first method and to develop an engineering complete theory. The forced response is given in the case of a concentrated harmonic excitation force with various intensities. The numerical results obtained here with the two formulations, using an explicit analytical solution, were compared with those obtained previously using a formulation in which the in-plane displacements have been neglected, showing an "over-stiff" effect.
\end{abstract}

\section{Introduction}

Some problems of plate vibrations cannot be adequately analysed using the linear theory of vibration, which is based on the assumption that the middle plane of the plate is inextensible and that the vibration amplitude is small, compared to the plate thickness. The design of structural elements, especially plates undergoing large vibration amplitudes due to high excitation forces, arising in modern aircraft and naval constructions, requires determination of accurate estimates, or at least estimates which are good enough from the safety point of view, of the dynamic characteristics, which are useful for predicting the dynamic responses in the real operational environment.

Geometrically non-linear vibrations of plates have been analysed by numerous researchers using analytical, numerical, or combined approximate methods [1-3]. In a previous series of papers, a semi-analytical model for geometrically non-linear free and forced vibrations of elastic thin straight structures, such as beams, homogeneous and symmetrically laminated rectangular plates has been developed by Benamar et al. [4]. The model was based on Hamilton's principle and spectral analysis, and was of the same spirit as the well-known Rayleigh-Ritz method, used for numerical approximate solution of linear problems of vibration.

This work is a complementary approach to investigate the geometrically non linear steady state periodic forced response of fully clamped rectangular plates (FCRP) excited by a concentrated force, taking into account, in addition to the transverse vibration, the in-plane displacements $\mathrm{u}$ and $\mathrm{v}$ in an average sense, in order to examine, using a simple practical engineering theory, the effect of the excitation force on the non-linear frequency response functions in the neighbourhood of the fundamental mode, corresponding to specified maximum amplitude of FCR plates.

The formulation proposed takes advantage of the immovable in-plane boundary conditions, to eliminate naturally the in-plane displacements $\mathrm{u}$ and $\mathrm{v}$, by neglecting the corresponding inertia in the non-linear amplitude equation. The expressions for the mass tensor $m_{i j}$, and the linear rigidity tensor $k_{i j}$ presented in [4,5] are unchanged, but the expression for the non-linear rigidity tensor $b_{i j k l}$ is affected in the present theory. Thus, the general features of the model remain the same and the mathematical treatment of the set of non-linear algebraic equations giving arise to solution of the problem of large vibration amplitudes of rectangular plates is carried out using an explicit analytical solution procedure proposed previously [6].

Fig.1. FCR plate notation, with a harmonic excitation force

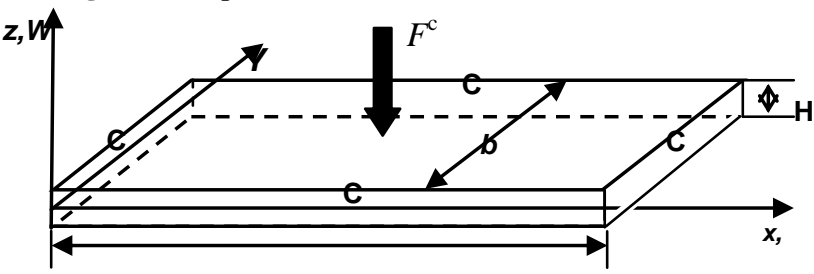




\section{The complete formulation}

To identify the displacement field $\mathrm{U}(\mathrm{x}, \mathrm{y}, \mathrm{t}), \mathrm{V}(\mathrm{x}, \mathrm{y}, \mathrm{t})$, and $\mathrm{W}(\mathrm{x}, \mathrm{y}, \mathrm{t})$ for a plate undergoing large transverse vibrations, the following Hamilton's function is used:

$\Phi(\omega)=\int_{0}^{2 \pi / \omega}\left(V_{t}-T\right) d t$

Where $V_{t}$ is the total strain energy which is given as the sum of the flexural strain energy $V_{f}$ and the axial strain energy induced by large deflections $V_{a}: V_{t}=V_{f}+V_{a} . T$ is the kinetic energy. The Von Kaman's non-linear straindisplacement relations for a rectangular plate are:

$\varepsilon_{x}^{m}=\frac{\partial u}{\partial x}+\frac{1}{2}\left(\frac{\partial w}{\partial x}\right)^{2} ; \quad \varepsilon_{y}^{m}=\frac{\partial v}{\partial y}+\frac{1}{2}\left(\frac{\partial w}{\partial y}\right)^{2} ; \quad \gamma_{x y}^{m}=\frac{\partial u}{\partial y}+\frac{\partial v}{\partial x}+\frac{\partial w}{\partial x} \frac{\partial w}{\partial y}$

$\varepsilon_{x}^{f}=-z \frac{\partial^{2} w}{\partial x^{2}} ; \varepsilon_{y}^{f}=-z \frac{\partial^{2} w}{\partial y^{2}} ; \gamma_{x y}^{f}=-2 z \frac{\partial^{2} w}{\partial x \partial y}$

In the above relations $\mathrm{u}(x, y, t)$ and $\mathrm{v}(x, y, t)$ are the in-plane displacements, whereas $w(x, y, t)$ is the transversal displacement of a point $(x, y)$ of the plate middle plane. The flexural strain energy expression, in the fully clamped boundaries case, is:

$$
V_{f}=\frac{1}{2} D \int_{S}\left(\frac{\partial^{2} W}{\partial x^{2}}+\frac{\partial^{2} W}{\partial y^{2}}\right)^{2} d S
$$

and the axial strain energy is given by:

$$
\begin{aligned}
V_{a}=\frac{E H}{2\left(1-v^{2}\right)} \int_{s}\left\{\left(\frac{\partial U}{\partial x}\right)^{2}+\left(\frac{\partial U}{\partial x}\right)\left(\frac{\partial W}{\partial x}\right)^{2}\right. & +\left(\frac{\partial V}{\partial y}\right)^{2}+\left(\frac{\partial V}{\partial y}\right)\left(\frac{\partial W}{\partial y}\right)^{2}+\frac{1}{4}\left[\left(\frac{\partial W}{\partial x}\right)^{2}+\left(\frac{\partial W}{\partial y}\right)^{2}\right]^{2} \\
& +2 v\left[\left(\frac{\partial U}{\partial x}\right)\left(\frac{\partial V}{\partial y}\right)+\frac{1}{2}\left(\frac{\partial V}{\partial y}\right)\left(\frac{\partial W}{\partial x}\right)^{2}+\frac{1}{2}\left(\frac{\partial U}{\partial x}\right)\left(\frac{\partial W}{\partial x}\right)^{2}\right] \\
+ & \left.\frac{1-v}{2}\left[\left(\frac{\partial U}{\partial y}\right)^{2}+2\left(\frac{\partial U}{\partial y}\right)\left(\frac{\partial V}{\partial x}\right)+\left(\frac{\partial V}{\partial x}\right)^{2}\right]\right\} d S
\end{aligned}
$$

The expression for the kinetic energy is given by:

$T=\frac{1}{2} \rho H^{2} \int_{S}\left[\left(\frac{\partial W}{\partial t}\right)^{2}+\left(\frac{\partial U}{\partial t}\right)^{2}+\left(\frac{\partial V}{\partial t}\right)^{2}\right] d S$

In which rotary inertia terms are neglected.

$D=\frac{E H^{3}}{12\left(1-v^{2}\right)}$ is the flexural stiffness of the plate. $\mathrm{H}$ and $\mathrm{S}$ are the plate thickness and area respectively. The material properties are the Young's modulus $E$, the Poisson's ratio $v$, and the mass per unit volume $\rho$.

\subsection{Solution procedure}

If the time and space functions are supposed to be separable and harmonic motion is assumed, the normal displacement function can be written as:

$$
W(x, y, t)=w(x, y) \sin \omega t
$$

Using the expressions for the axial displacements commonly adopted [6-8], the corresponding functions $U(x, y, t)$ and $V(x, y, t)$ are assumed to be:

$$
U(x, y, t)=u(x, y) \sin ^{2} \omega t ; \quad V(x, y, t)=v(x, y) \sin ^{2} \omega t
$$

Expanding $\mathrm{u}(\mathrm{x}, \mathrm{y}), \mathrm{v}(\mathrm{x}, \mathrm{y})$, and $\mathrm{w}(\mathrm{x}, \mathrm{y})$ in the form of finite series, involving implicit summation for repeated indices:

$$
\begin{aligned}
& u(x, y)=b_{i} u_{i}(x, y) \\
& v(x, y)=c_{j} v_{j}(x, y) \\
& w(x, y)=a_{k} w_{k}(x, y)
\end{aligned}
$$

Substituting expressions (8-10) into equation (2-4) and rearranging, the discretised expressions for $V_{f}, \mathrm{~V}_{\mathrm{a}}$, and $T$ can be determined. Hamilton's principle states:

$$
\delta \Phi=\delta \int_{0}^{2 \pi / \omega}\left(V_{t}-T\right) d t=0
$$

The application of equation (11) reduces the problem to that of finding the minimum of the functional $\Phi$ with respect to the $a_{i}$ 's, which leads to three sets of non linear algebraic equations, written in a non-dimensional form as follows:

$$
\left\{\begin{array}{lr}
3 a_{i} a_{j} a_{k} b_{i j k r}^{1^{*}}+2 a_{i} a_{j} k_{i j}^{1^{*}}+\frac{3}{2} b_{i} a_{j} n_{i j r}^{1^{*}}+\frac{3}{2} c_{i} a_{j} n_{i j r}^{2^{*}}-2 \omega^{*^{2}} a_{i} m_{i r}^{1^{*}}=0 \\
r=1, \ldots n \\
\frac{3}{2} b_{i} k_{i t}^{2^{*}}+\frac{3}{4}\left(c_{j} k_{i j}^{4^{*}}+a_{j} a_{k} n_{t i k}^{1^{*}}\right)-2 \lambda^{2} \omega^{*^{2}} b_{i} m_{i t}^{2^{*}}=0 & t=1, \ldots, m \\
\frac{3}{2} c_{i} k_{i s}^{3^{*}}+\frac{3}{4}\left(b_{i} k_{i s}^{4^{*}}+a_{j} a_{k} n_{s j k}^{i^{i}}\right)-2 \beta^{2} \omega^{*^{2}} c_{i} m_{i s}^{3^{*}}=0 & s=1, \ldots, m
\end{array}\right.
$$

Since $\lambda^{2}$ and $\beta^{2}$ are very small quantities for thin plates, the terms involving these two parameters in equations $(13,14)$, which actually correspond to in-plane inertia, can be neglected, which leads, after substituting and rearranging, to:

$$
3 a_{i} a_{j} a_{k} b_{i j k r}+2 a_{i} a_{j} k_{i j}^{i^{*}}-2 \omega^{*^{2}} a_{i} m_{i r}^{i^{*}}=0 \quad r=1, \ldots, n
$$

Equation (15) involves a new fourth non-linear rigidity tensor $b_{i j k l}^{1^{*}}$ the expression of which depends on the tensors $b_{i j k l}^{1^{*}}, n_{i j k}^{1^{*}}, n_{i j k}^{2^{*}}, k_{i j}^{2^{*}}, k_{i j}^{3^{*}}$, and $k_{i j}^{4^{*}}$.

\section{The averaging technique}

Consider the plate shown in Figure 1. Its total strain energy $\mathrm{V}$ is the sum $V f+V_{a}$, where $V_{f}$ is defined by [7]:

$$
V_{f}=\frac{1}{2} \int_{S} \frac{E}{2\left(1-v^{2}\right)}\left(\varepsilon_{x}^{f^{2}}+\varepsilon_{y}^{f^{2}}\right)+\frac{E}{4(1+v)} \gamma_{x y}^{f^{2}} d S
$$


The expression for the membrane strain energy $V_{a}$, which is due solely to the stretching of the middle surface of the plate, is given by [7]:

$$
V_{m}=\frac{E h}{2\left(1-v^{2}\right)} \int_{S}\left(\varepsilon_{x}^{m^{2}}+\varepsilon_{y}^{m^{2}}+2 v \varepsilon_{x}^{m} \varepsilon^{m}+\frac{1}{2}(1-v) \gamma_{x y}^{m^{2}}\right) d S
$$

The averaged normal non-linear membrane strains $\left(\varepsilon_{x}^{m}\right)_{a}$ and $\left(\varepsilon_{y}^{m}\right)_{a}$, defined below, at a point of coordinates $\left(\mathrm{x}_{0}, \mathrm{y}_{\mathrm{o}}\right)$ of the plate middle plane, are used herein to determine the expression for the associated membrane strain energy $\left(V_{m}\right)_{\mathrm{a}}$. This is the basic idea of the $\mathrm{u}-\mathrm{v}-\mathrm{w}-$ average formulation.

$\left(\varepsilon_{x}^{m}\right)_{a}\left(y_{o}\right)=\frac{1}{a} \int_{0}^{a} \varepsilon_{x}^{m} d x$ and $\left(\varepsilon_{y}^{m}\right)_{a}\left(x_{o}\right)=\frac{1}{b} \int_{0}^{b} \varepsilon_{y}^{m} d y$

Then,

$$
\begin{aligned}
& \left(\begin{array}{c}
\varepsilon^{m} \\
x
\end{array}\right)_{a}\left(y_{o}\right)=\frac{1}{a}\left[\left.\int_{0}^{a} \frac{\partial u}{\partial x}\right|_{y=y_{o}} d x+\left.\frac{1}{2} \int_{0}^{a}\left(\frac{\partial W}{\partial x}\right)^{2}\right|_{y=y_{o}} d x\right] \\
& \left(\begin{array}{c}
\varepsilon^{m} \\
y
\end{array}\right)_{a}\left(x_{o}\right)=\frac{1}{b}\left[\left.\int_{0}^{b} \frac{\partial v}{\partial y}\right|_{x=x_{o}} d y+\left.\frac{1}{2} \int_{0}^{b}\left(\frac{\partial W}{\partial y}\right)^{2}\right|_{x=x_{o}} d y\right]
\end{aligned}
$$

Being concerned here only with immovable edge conditions, the first terms appearing in the above averaged membrane strain expressions vanish, i.e.:

$$
\int_{0}^{a} \frac{\partial u}{\partial x} d x=\int_{0}^{b} \frac{\partial v}{\partial y} d y=0
$$

On the other hand, terms involving the in-plane displacements of the non-linear shear strain $\gamma_{x y}$ are neglected, i.e.:

$\gamma_{x y} \approx \frac{\partial W}{\partial x} \frac{\partial W}{\partial y}$

Thus, the expression for the averaged membrane strain energy $\left(V_{m}\right)_{a}$ is given by:

$$
\begin{aligned}
& \left(V_{m}\right)_{a}=\frac{E h}{2\left(1-v^{2}\right)}\left\{\frac{1}{4 a} \int_{0}^{b}\left[\left.\int_{0}^{a}\left(\frac{\partial W}{\partial x}\right)^{2}\right|_{y=y_{o}} d x\right]^{2} d y+\frac{1}{4 b} \int_{0}^{a}\left[\left.\int_{0}^{b}\left(\frac{\partial W}{\partial y}\right)^{2}\right|_{x=x_{o}} d y\right]^{2} d x+\right. \\
& \frac{2 v}{4 a b} \int_{0}^{b}\left[\left.\int_{0}^{a}\left(\frac{\partial W}{\partial x}\right)^{2}\right|_{y=y_{o}} d x\right] d y \int_{0}^{a}\left[\left.\int_{0}^{b}\left(\frac{\partial W}{\partial y}\right)^{2}\right|_{x=x_{o}} d y\right] d x+ \\
& \left.\frac{1-v}{2} \int_{0}^{a} \int_{0}^{b}\left(\frac{\partial W}{\partial x}\right)^{2}\left(\frac{\partial W}{\partial y}\right)^{2} d x d y\right\}
\end{aligned}
$$

This averaging expression for the axial strain energy is the main novelty of the so-called averaging formulation proposed.

\section{Case of FCR plates excited harmonically by a concentrated force}

Consider the geometrically non-linear steady state periodic forced response of a FCRP, excited harmonically by a concentrated force. The dimensional generalized force $f_{i}^{c}$ corresponding respectively to the concentrated force $F^{c}$ at point $\left(\mathrm{x}_{0}, \mathrm{y}_{0}\right)$ for a fully clamped rectangular plate having the characteristics a, b, H, and D, is [6]:

$f_{i m}{ }^{c^{*}}=\frac{b^{3} F^{c}}{a D H} \phi_{i}^{*}\left(x_{0}^{*}, y_{0}{ }^{*}\right)$

A forcing vector $\left\{F^{*}(t)\right\}$ is defined by:

$F_{i m}{ }^{c}(t)=F^{c} \phi_{i}^{*} \delta\left(x_{0}, y_{0}\right) \sin (\omega t)=f_{\text {im }}{ }^{c} \sin (\omega t)$

Adding the forcing term to the right hand side of equations (15) written in the modal function basis for complete and averaging technique leads respectively to:

$$
\begin{aligned}
& 3 a_{i} a_{j} a_{k} b_{i j k r}^{*}+2 a_{i} a_{j} k_{i j}^{1^{*}}-2 \omega^{* 2} a_{i} m_{i r}^{1^{*}}=2 f_{i m}^{*} \quad i=1, \ldots, n \\
& 3 a_{i} a_{j} a_{k} b_{i j k r}^{(a)}+2 a_{i} k_{i j}-2 \omega^{2} a_{i} m_{i r}=2 f_{i m} \quad r=1, \ldots, n
\end{aligned}
$$

This non-linear algebraic system, corresponding to the non-linear steady state periodic forced response of a FCRP, The frequency parameter $\omega^{*}$ represents the excitation frequency, which varies in the range chosen for performing the excitation tests, or for examining theoretically the nonlinear forced response.

\section{Multimode approach, in the vicinity of the first non-linear mode.}

Applying the explicit solution procedure proposed previously in [6] to equations (26) and (27) leads to:

$\left(k_{r r}^{1^{*}}-\omega^{*^{2}} m_{r r}^{*}\right) a_{r}+\frac{3}{2} a_{1}^{3} b_{111 r}^{*}=f_{r m}^{*} \quad r=2 \ldots n$

Then, the modal contributions $\varepsilon_{i}$ for $i=2, \ldots, n$ of the symmetric basic functions corresponding to a given value of the assigned first basic function contribution $a_{1}$ are explicitly obtained as follows:

$\varepsilon_{r}=\frac{f_{r m}{ }^{*}-\frac{3}{2} a_{1}^{3} b_{111 r}^{*}}{k_{r r}^{*}-\omega^{2^{*}} m_{r r}^{*}} \quad r=2 \ldots n$

The first harmonic component of the non-linear steady state periodic forced response function $W_{n l l}\left(x^{*}, y^{*}, a_{1}\right)$, for a given assigned value of $a_{l}$, is defined with an explicit simple formula as : 


$$
\begin{aligned}
& W_{n l}^{*}\left(x, y, a_{1}\right)=\frac{f_{1 m}^{i}-\frac{3}{2} a_{1}^{3} b_{1111}^{1^{*}}}{\left(k_{11}^{1^{*}}-\omega^{* 2} m_{11}^{1 *}\right)} \phi_{1}^{*}\left(x^{*}, y^{*}\right)+\frac{f_{2 m}^{i^{*}}-\frac{3}{2} a_{1}^{3} b_{2111}^{1^{*}}}{\left(k_{22}^{1 *}-\omega^{* 2} m_{22}^{1 *}\right)} \phi_{3}^{*}\left(x^{*}, y^{*}\right)+\ldots \\
& \ldots \ldots . .+\frac{f_{9 m}^{i}-\frac{3}{2} a_{1}^{3} b_{9111}^{1^{*}}}{\left(k_{99}^{1 *}-\omega^{* 2} m_{99}^{1 *}\right)} \phi_{9}^{*}\left(x^{*}, y^{*}\right)
\end{aligned}
$$

This expression permits determination of the distribution of the associated non-linear bending stress in the FCRP, under a harmonic force, with immovable edge conditions. To determine the distribution of the associated membrane stress, the in-plane displacement coefficients $b_{i}$ and $c_{i}(i=$ $1, \ldots, m)$ have to be determined as function of $a_{l}$ and the $\varepsilon_{i}$ 's for $i=2, \ldots, n$.

\section{Numerical details}

The averaged formulation described in this work has been applied to isotropic fully clamped rectangular plates with various aspect ratios, with all edges immovable. Steel plates have been considered with material properties: Young's modulus of elasticity E $=200 \mathrm{GPa}$, Poisson's ratio $v=0.33$, and material density $\rho=7850 \mathrm{Kg} / \mathrm{m} 3$.

Beam functions have been used as in many previous studies on linear and non-linear vibrations of FCRP. The parameters $b_{i j k l}{ }^{*}, k_{i j}{ }^{*}$ and $m_{i j}{ }^{*}$ were computed numerically. In-plane displacement functions have a strong influence on the geometrically non-linear dynamic property of rectangular plates (Figure 2 and Figure 3).

Fig. 2. The forced response of a FCRP $(\alpha=1)$ excited by a harmonic concentrated force $\left(F^{c^{*}}=200\right)$.

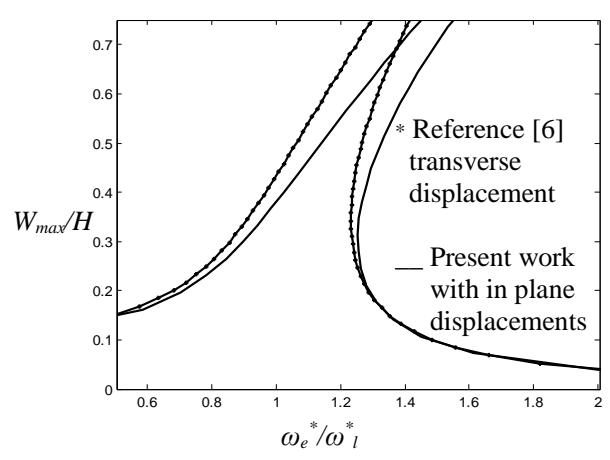

Fig. 3. The forced response of a FCRP $(\alpha=1)$ excited by harmonic concentrated force $F^{c^{*}}=100, F^{c^{*}}=250$ and $F^{c^{*}}=300$

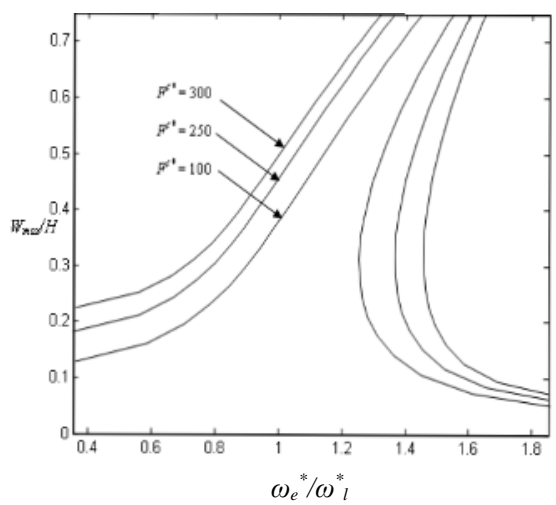

\section{Conclusions}

The geometrically non-linear steady state periodic forced response of FCR plates excited by a concentrated force has been studied using an averaging technique, based on the assumption that the in-plane membrane strains are constant over the whole span of the plate with immovable edges, which allows them to be averaged before being substituted in the associated energy integral. On the other hand, the average formulation consists on estimating the membrane stresses by considering the deflection obtained from the solution of the formulation, previously used. Another feature of the present work is to determine the effect of the excitation frequency and level of the applied harmonic force, concentrated at the plate middle span, on its dynamic response at large vibration amplitudes.

\section{References}

1. G. Prathap. Comments on "effect of longitudinal or in-plane deformation and inertia on the large amplitude flexural vibrations of beams and thin plates. Journal of Sound and Vibration, 55, 308-311. (1977)

2. B. Nageswara Rao. Large-amplitude free vibrations of simply supported uniform beams with immovable ends. Journal of Sound and Vibration 155, 523-527 (1992)

3. R. Lewandowski. Free vibration of structures with cubic non-linearity remarks on amplitude equation and Rayleigh quotient. Journal of Sound and Vibration 192, 1681-1709. (2003)

4. R. Benamar, M.M.K. Bennouna and R. G. White. The effects of large vibration amplitudes on the fundamental mode shape of thin elastic structures, part II: Fully clamped rectangular isotropic plates. Journal of Sound and Vibration 164, 295-316. (1993)

5. K. EL Bikri, R. Benamar and M.M Bennouna Geometrically non-linear free vibrations of clamped simply supported rectangular plates. Part I: the effects of large vibration amplitudes on the fundamental mode shape. Computers \& Structures, 81, 2029-2043. (2003)

6. M. EL Kadiri, R. Benamar and R. G. White. Improvement of the semi-analytical method, based on Hamilton's principle and spectral analysis, for determination of the geometrically non-linear free response of thin straight structures. Part III: steadystate periodic forced response of simply supported and fully clamped rectangular plates. Journal of Sound and Vibration 264, 1-35. (2003)

7. S.P. Timoshenko, SW. Krieger, second edition. Theory of plates and shells. McGraw-Hill International student edition. (1959). 